De Tampere à Séville : bilan de la sécurité européenne $(1 / 2)$

\title{
De Tampere à Séville, vers une ultra gouvernementalisation de la domination transnationale?
}

From Tampere to Seville, toward Excessive Government in Transnational Leadership

Didier Bigo et Elspeth Guild

\section{(2) OpenEdition}

Édition électronique

URL : http://journals.openedition.org/conflits/762

DOI : $10.4000 /$ conflits.762

ISSN : $1777-5345$

Éditeur :

CCLS - Centre d'études sur les conflits lilberté et sécurité, L'Harmattan

\section{Édition imprimée}

Date de publication : 1 mars 2002

Pagination : 5-25

ISBN : 2-7475-3029-9

ISSN : 1157-996X

\section{Référence électronique}

Didier Bigo et Elspeth Guild, «De Tampere à Séville, vers une ultra gouvernementalisation de la domination transnationale? », Cultures \& Conflits [En ligne], 45 | printemps 2002, mis en ligne le 22 mars 2006, consulté le 30 mars 2021. URL : http://journals.openedition.org/conflits/762 ; DOI : https://doi.org/10.4000/conflits.762

Ce document a été généré automatiquement le 30 mars 2021.

Creative Commons License 


\title{
De Tampere à Séville, vers une ultra gouvernementalisation de la domination transnationale?
}

\author{
From Tampere to Seville, toward Excessive Government in Transnational \\ Leadership
}

Didier Bigo et Elspeth Guild

1 De Tampere à Séville, nous avons entamé une crise en spirale de la représentation politique allant des espoirs des ONG dans Tampere aux désillusions de Laeken et Séville, en passant par la colère de l'après-Gênes. Cette crise engendre une légitimité toujours plus chancelante de l'Union ainsi qu'une délégitimation forte des décisions des sommets du G8. Mais cela semble paradoxalement renforcer la détermination des divers gouvernants à imposer leurs vues sur la sécurité et l'ordre mondial. Dès lors, la période contemporaine est riche d'événements divers scandés par les noms de villes des sommets, Tampere, Québec, Nice, Göteborg, Gênes, Laeken, Séville... et une date le 11 septembre, qui n'ont quasiment rien en commun en termes institutionnels, en termes de discussion sur des sujets particuliers, mais qui pourtant font sens ensemble.

2 Ce sens est celui d'une ultra-gouvernementalisation de la domination transnationale. Il a comme soubassement une transformation des modalités politiques de la domination et de l'émancipation du politique du cadre étatique (et interétatique). En effet, alors que l'on assiste à une accélération sans précédent de la transnationalisation de la gouvernance à l'échelle mondiale, tout est fait par les autorités pour qu'il ne puisse émerger une transnationalisation de la contestation créant un espace public de même dimension que celui de la gouvernance qui s'institue à coup de normes, de soft law et de zones d'exception où les droits des individus sont suspendus au profit des règlements administratifs. C'est dans ce décalage remettant en cause les fondements de la démocratie entre une "administration" mondialisée de la vie collective et des échanges sociaux et économiques d'une part, et la tentative de blocage par cette même administration de la contestation transnationale que s'inscrivent l'ensemble des questions institutionnelles créant de nouvelles arènes de discussion, l'analyse des 
trajectoires des mouvements sociaux vers la violence ou non, l'examen de la légalité des pratiques policières ainsi que des stratégies et des tactiques d'(in)sécurisation des gouvernants à l'égard des contestataires... ${ }^{1}$

3 Cette transnationalisation de la gouvernance - pour reprendre momentanément le terme en usage dans les institutions et les cabinets d'expertise - est loin d'être chose nouvelle. La sphère de l'économie politique internationale a analysé la montée en puissance des firmes multinationales et leurs capacités comme acteurs politiques à imposer aux politiciens traditionnels des modalités de régulation favorisant le développement d'une certaine forme de capitalisme les favorisant directement comme " héritiers » d'un système et limitant l'accès des "prétendants » obligés de recourir à des formes plus primitives d'accumulation du capital ${ }^{2}$. Les pays développés en ont profité contre les pays en voie de (sous)développement. L'OMC, en institutionnalisant des règles du jeu favorables au capital financier sur la production, a encore plus favorisé la vitesse de circulation des capitaux, des services sur les biens et les personnes et a encouragé la rentabilité à court terme des opérations sur les investissements à long terme. Les acteurs privés ont pris un rôle politique qui a depuis longtemps entamé la prétention des gouvernants élus d'avoir le poids le plus effectif sur les politiques économiques nationales et ils ont réduit le keynésianisme à une réflexion quasi obsolète. Derrière le terme de "forces anonymes du marché ", on peut déceler des acteurs puissants dont les grandes banques et les firmes multinationales d'origine occidentale. La mondialisation économique dans son orientation néo-libérale est de leur fait, et n'est en rien une tendance inéluctable.

4 Les dirigeants des grands Etats ont symboliquement voulu, en se réunissant en "Sommets", montrer qu'ils continuaient de diriger et que leurs déclarations communes orientaient les "marchés ». Il en va, pensent-ils, de la crédibilité et de l'avenir des « professionnels » de la politique à l'égard de leurs électeurs. Comme on ne sait pas qui élit les banquiers, c'est vers les gouvernements que se tournent les demandes, même si une certaine désaffection des élections et la montée des extrémisme montrent que le doute sur les capacités d'action des hommes politiques élus ne cesse d'augmenter. La production de déclarations, de recommandations, de programmes d'action s'est donc multipliée et s'est diversifiée pour réaffirmer symboliquement que le centre de décision est toujours tenu par les gouvernements les plus puissants. A la dimension de politique économique des sommets comme le G7, s'est adjoint rapidement une dimension de politique (a)sociale puis une dimension de politique (in)sécuritaire. Ces sommets qui devaient «améliorer la croissance» ont tourné à une série de prescriptions contre les Etats bloquant le jeu du marché dans les pays du Sud et à des discours unanimistes condamnant toutes les formes de violence affectant les pays occidentaux (puis la Russie). Le G7-G8 de Lyon a légitimé le retour de la Russie dans le club des « grands » et son attitude à l'égard de la Tchétchénie, surtout depuis le 11 septembre.

5 Les réunions ont scandé depuis quelques années les routines administratives des divers ministères des affaires étrangères en fonction des «agendas » retenus pour la prochaine réunion. Un certain nombre de priorités ont été instaurées en fonction de ces discussions, marginalisant le rôle des parlements nationaux, là où ils ont encore une quelconque influence sur les politiques gouvernementales. Cet espace élargi de gouvernance que symbolise le G7-G8 a été de plus en plus «mis en scène » comme « spectacle politique » convoquant les « grands de ce monde » et son rôle s'est renforcé 
après le 11 septembre, mais sa visibilité plus grande a aussi entraîné une contestation de plus en plus significative avec la constitution de "contre-sommets $»^{3}$. Une contestation populaire transnationale (popular dissent) s'est forgée depuis Seattle et a largement dépassé le cadre de petits groupes manifestants radicalisés. Même si ces derniers existent (et sont instrumentalisés par les gouvernements et leurs polices comme à Gênes), la contestation est bien plus large. Elle s'appuie sur la distanciation toujours plus grande du mandat représentatif et le fait que les gouvernants voudraient n'avoir aucun compte à rendre entre les élections, même lorsqu'ils agissent $a$ contrario du programme sur lequel ils ont été élus.

6 Avec l'institutionnalisation du cadre européen à travers l'Union Européenne au-delà du simple marché commun, on a constaté à la fois le même mouvement de fond, réduisant les discussions publiques et politiciennes au profit de discussions discrètes, voire confidentielles entre les administrations et les parties les plus puissantes des secteurs privés et para-publics, et des résistances par le droit de plus en plus fortes. On a écarté ou minoré les procédures parlementaires dans la plupart des pays de l'Union, en laissant les gouvernements discuter entre eux. Le dialogue intergouvernemental et surtout les dialogues sectoriels entre ministères de même type des différents pays européens ont pris le pas sur les discussions interministérielles nationales. Les positions communes nationales ont eu de moins en moins d'impact dans les décisions d'infrastructure et de long terme et ont été de plus en plus cantonnées à la gestion des dossiers de politique symbolique. Des « archipels » différents se sont constitués, reliant les secteurs bancaires des divers pays, les divers ministères du travail et certaines grandes entreprises, les institutions militaires, policières, judiciaires ou pénales en construisant des lignes d'opposition d'intérêts professionnels transcendant les clivages nationaux et réduisant ces derniers à n'être que des luttes secondaires - mais médiatisées ${ }^{4}$. Cette transnationalisation des archipels bureaucratiques a cassé la notion même de gouvernement au sens classique du terme. Elle a plus à voir avec l'ordre de la gouvernementalité foucaldienne qu'avec des formes de gouvernance, fussent-elles à plusieurs niveaux. Ce sont des technologies transversales de pouvoir qui semblent orienter les décisions et non des prises de parole politiques. Les hommes politiques semblent de moins en moins présents dans les décisions de l'Union malgré leurs efforts rhétoriques. La gouvernementalité «chasse» le politique et le recouvre. Au décisionnisme extrême qui prend forme après le 11 septembre correspond, non pas une réduction du nombre de décideurs, mais au contraire une anonymisation de ceux-ci, une déresponsabilisation que ne cache pas l'hyper personnalisation de la politique par les grands dirigeants. La notion d'appartenance citoyenne (nationale ou européenne) en est fragilisée.

7 Les sommets de l'Union ont donc été associés en partie avec ceux du G7-G8. On a vu néanmoins avec l'européanisation la mise en place de mécanismes de droit et de recours des citoyens limitant les espaces ouverts à la domination transnationale. La présence d'un Parlement européen, d'un ombudsman, de la Cour de justice des communautés et de la Cour européenne des droits de l'Homme ont structuré au niveau européen un espace de «négociation » des identités et des projets, obligeant à une prise en compte d'intérêts plus divers ${ }^{5}$. Il n'a pas été possible dans le cadre européen d'éviter le débat sur les libertés civiles que le G7 et l'OMC avaient réussi à circonvenir. Des ONG, des associations, des syndicats ont été marginalement associés à la construction de cet espace élargi de gouvernance de l'UE qui ne répond certes plus aux caractéristiques de l'Etat-Nation frontaliérisé (théorisé par Max Weber), mais qui s'institue aussi contre la 
dominance économique néo-libérale du G7 et qui y puise dès lors des ressources de légitimation. Le rôle du droit européen, d'un espace où les individus comme sujets de droit peuvent demander des comptes à une Cour qui ne dépend pas de leur Etat contre ce dernier ou contre d'autres Etats, concoure à imposer certaines formes de «responsabilité » et de "transparence» qui ne sont pas équivalentes à une réelle démocratisation mais qui contrebalancent la faible représentation politique.

8 L'originalité d'une institution comme la Commission (qui n'est guère le gouvernement d'un futur Etat européen) a été de créer des espaces de tension et des arènes où l'unanimité au sein du Conseil ne suffisait pas à imposer une norme, si celle-ci était frontalement en opposition avec l'ensemble des institutions représentant le pôle du droit et des droits ${ }^{6}$. Les combats pour la transparence des décisions, les luttes contre l'américanisation de l'Union via le refus d'une Otanisation des deuxième et troisième piliers, et d'autres luttes encore dans le domaine des libertés civiles, sont des exemples de ces «mises en tension » que ce numéro exemplifie avec la question de la directive contre le racisme, la question du droit d'asile, celle du mandat d'arrêt européen et de la création d'un espace judiciaire.

La séparation en termes de piliers instituée avec Maastricht avait été le moment d'un compromis, ayant peu de rapports avec les vieux argumentaires de souveraineté versus fédéralisme, entre une "gouvernementalisation » complète de la domination avec un risque d'arbitraire et de "pouvoir nu» selon les lignes du G8 et de l'américanooccidentalisation, et une "parlementarisation-commissionisation » insufflant au sein des espaces de négociation des éléments de discussion des parties dominées des sociétés civiles en contrepartie d'une acceptation plus grande de ces transformations. Les réseaux de police et de services de renseignement s'étaient saisis des questions de l'immigration mais aussi de l'asile et du franchissement des frontières et, via Schengen avaient imposé une certaine vision de ces évolutions mondiales en termes de problèmes de sécurité. On avait vu alors un Parlement européen combatif et allié souvent avec la Commission et les acteurs du premier pilier.

10 Avec Amsterdam redéfinissant les limites des premier et troisième piliers en faveur du premier, et ensuite avec les déclarations de Tampere on avait pu croire un moment que, concernant certains secteurs comme la politique sociale, le droit des étrangers résidant sur le territoire de l'Union, l'antiracisme, l'ouverture rapide vers les PECO, cette deuxième ligne de force s'imposait ${ }^{7}$. Lors du Sommet de Tampere en octobre 1999, l'Union européenne avait en effet dévoilé son plan de réalisation d'une zone de liberté, de sécurité et de justice promise par les amendements du Traité d'Amsterdam apportés au traité de l'Union européenne. Les ressources cumulées du droit et de l'activisme de certaines ONG commençaient à enserrer dans leur régulation, les modalités de la gouvernementalité transnationale, au moins à l'échelle européenne de l'Union. La création d'une charte, vue par certains comme une future constitution, l'élargissement des possibilités d'action du judiciaire à l'échelle européenne, indépendamment des bonnes volontés des hommes politiques, l'investissement - au moins discursif - dans un « espace public citoyen » auxquels tous les individus vivant sur le territoire de l'Union pourraient virtuellement intervenir, remettaient la démocratie sous forme néoparlementaire au goût du jour européen. Le renforcement d'une domination symbolique évitant la mise à nue des rapports de force et passant par la croyance dans la démocratie des élites non économiques et un renforcement du pôle juridique - et donc de leurs propres ressources - semblait accroître les possibilités de légitimation. 
11 Mais la crise conjoncturelle de la Commission ${ }^{8}$, la réaction des gouvernements à cette tendance renforçant les contraintes juridiques à leur égard, leurs pratiques à Nice à l'égard de la Commission, la mise sur pied d'une Europe militaire et policière pouvant intervenir hors des frontières de l'Union et finalement les stratégies d'inter-pilarisation ont produit des effets inversant la tendance. Ils se sont traduits, non par un renforcement de la Commission et du droit européen comme l'espéraient certains lors de Tampere, mais au contraire par une «schengenisation» du premier pilier, et par le renforcement à son détriment des deuxième et troisième piliers. L'Union a été fragilisée dans son ensemble par ces conflits internes, par les luttes inter-piliers et par le manque de cohérence des personnels la représentant à l'étranger. Pour ne prendre qu'un exemple, les relations entre Solana et Prodi n'ont jamais été véritablement clarifiées en ce qui concerne la discussion avec les Etats-Unis. Le secrétariat du Conseil s'est mis à concurrencer la Commission dans certains domaines, tout en menant une politique qui ne convenait pas forcément aux désirs des Etats. Les deuxième et troisième piliers ne trouvaient guère leurs marques réciproques et au sein du troisième pilier le Commissaire Vittorino a eu beaucoup de mal - et en a toujours - pour imposer ses thèses à l'égard de son directeur Fortescue. Seule une volonté d'équilibrage à l'égard des Etats-Unis maintenait une certaine cohérence au sein de l'Union.

12 Seulement, après le 11 septembre, les Etats-Unis ont renforcé leur pression et sont devenus maintenant un acteur clé de l'européanisation via leurs demandes auprès de la Commission concernant les domaines du renseignement, de la coopération policière et judiciaire, de la politique étrangère et insensiblement du franchissement des frontières et du marché commun'. Ils ont déstabilisé le rapport de forces entre sécurité coercitive d'une part (armée, police, justice), et sécurité comme garantie juridique et garantie sociale d'autre part en ne mettant l'accent que sur la première forme de sécurité. Ils ont voulu réactiver un " périmètre de sécurité » (traduit par le homeland security) fondé sur la souveraineté et la territorialité dans lequel l'Union ferait figure de premier front, mais ceci au détriment de la cohérence de l'Union qui repose depuis longtemps sur des mises en réseaux et sur des équilibres de ressources, bien plus que sur un principe territorial fixe. Actuellement, la conception autoritaire de la sécurité qu'ont forgée les Etats-Unis avec le gouvernement Bush s'est imposée progressivement contre l'idée d'une sécurité plus globale, non militaire que proposaient certains membres de l'Union et surtout certaines institutions transnationales, ainsi que certains démocrates américains. Ceci est peut-être conjoncturel. Les déclarations de changements sont pour l'instant plus importantes que les changements effectifs. Néanmoins il ne s'agit pas uniquement de politique symbolique. Les administrations sont restructurées, les budgets sont mis en place, les droits des étrangers sont minorés ou ignorés.

Laeken et Séville ont renforcé au niveau européen cette pente autoritaire venue d'outre-Atlantique, aussi bien au sein du Conseil qu'au sein de la Commission. Les discours sur la libre circulation des personnes, sur la citoyenneté européenne pour tous les individus vivant en Europe (y compris les extra-communautaires) ont été minorés et battus en brèche par les discours sur la menace terroriste post 11 septembre. Les étrangers et leurs enfants ont de nouveau été associés comme dans le milieu des années 1980 à des menaces contre les identités nationales, le welfare et la sécurité ${ }^{10}$. Les gouvernements américain et britannique ont choisi la suspension de certains droits fondamentaux au nom de la lutte antiterroriste et de la nécessité de mesures d'exception temporaires. D'autres gouvernements ont été moins explicites, mais ils ont 
choisi aussi dans leurs pratiques de renforcer les logiques de contrôle sur les personnes avec une "re-sacralisation des frontières extérieures" comme "ligne de front militaire » face aux « invasions » et «infiltrations terroristes » (et Séville en a été une preuve après le signe avant-coureur de Laeken).

En décembre 2001, au Sommet de Laeken, l'UE avait dû montrer jusqu'où elle était allée dans la réalisation des objectifs de Tampere. On s'était rendu compte immédiatement de l'échec de la libéralisation voulue à Tampere (si tant est qu'elle était voulue et n'était pas purement rhétorique). Le Conseil en a été réduit à trouver un accord sur des mesures répressives contre les réfugiés et non sur des mesures positives visant à garantir les obligations internationales de la protection. Dans le domaine de l'asile, seules trois mesures ont été prises: le financement du fonds européen pour les réfugiés, le règlement créant Eurodac (un fichier d'empreintes génétiques des demandeurs d'asile et des étrangers en situation irrégulière) ainsi qu'une directive sur la protection temporaire. L'objectif de rapprocher les droits du long séjour pour les résidents d'Etats tiers au sein de l'Union des droits dont jouissent les citoyens de l'Union n'a rencontré aucun succès. Au moment où les conclusions du Sommet de Laeken ont été tracées, aucune référence aux résidents de longue durée d'origine extracommunautaire n'était faite. La protection et l'extension des droits du long séjour pour les ressortissants d'Etats tiers ont été rayées de l'agenda, en dépit de l'importance et de l'accent mis sur cette question deux ans plus tôt et du travail de proposition d'une législation issue de la Commission. En revanche, sur la question de l'immigration illégale, non seulement trois mesures ont été adoptées (reconnaissance mutuelle des décisions d'expulsion, sanctions envers les transporteurs, financement du Système d'Information Schengen II), mais bien d'autres sont sur la table des négociations, car ce thème est devenu le point central des conclusions de Laeken: une action plus importante à l'encontre des immigrés illégaux, un accroissement des accords de réadmission avec les Etats tiers qui les obligent à accepter le retour de leurs ressortissants, une multiplication des mesures de police visant à lutter contre le trafic d'êtres humains. Et Séville n'a fait qu'accentuer ces mesures, ceci en dépit du fait qu'il y a bien peu d'indices d'un niveau substantiel d'organisation criminelle en ce qui concerne les "passeurs» d'êtres humains eux-mêmes, et que ce trafic, aussi répréhensible soit-il, semble plutôt se réduire à une activité artisanale dans certaines zones frontalières ${ }^{11}$. A Tampere, le Conseil avait par ailleurs promis un meilleur accès à la justice en Europe, étant étendu qu'on y arriverait par une "reconnaissance mutuelle renforcée des décisions judiciaires et des jugements et le nécessaire rapprochement des législations» mais la manière dont Europol et Eurojust fonctionnent actuellement depuis le 11 septembre, Laeken et Séville ne va nullement dans le sens d'un équilibre de la justice mais bien plus dans celui d'une coordination des "accusations $»^{12}$. Alors que les conclusions de Tampere précisent par exemple les principes d'un procès équitable et la délicate question de la collecte et de l'échange de preuves, et évoquent le «corpus juris ", les conclusions de Laeken notent avec satisfaction la création d'Eurojust sous forme d'un réseau institutionnel des procureurs nationaux ayant pour objectif de simplifier et accélérer la coopération, la coordination et les enquêtes d'« actes ", de conserve avec Europol. Eurojust permet alors un vaste échange d'informations, y compris des données personnelles sujettes avant tout au droit national. Dès lors la décision rend le «jurisdiction shopping " possible pour les parquets travaillant avec Europol. Il se pourrait alors que les procureurs les plus cyniques analysent les informations qu'ils reçoivent d'Europol, décident dans quel pays un procès équitable 
peut s'achever par une condamnation impossible ailleurs et requièrent alors que les accusations criminelles soient apportées dans ces mêmes pays alors que les défenseurs ne pourront agir avec la même possibilité. Le résultat des travaux dans le domaine de la coopération entre police et justice pour les affaires pénales consiste finalement à travers Laeken et Séville en une transformation du pouvoir de punir au sein de l'Union. Ce pouvoir a été central pour les autorités étatiques - le choix de punir ou non étant une décision politique à tous les niveaux de l'autorité judiciaire et policière. Maintenant, le pouvoir de punir trouve sa réalisation dans la dimension européenne. C'est ici que les événements de Göteborg et Gênes deviennent centraux dans cette transformation. La construction de ces manifestations de jeunes comme constituant la menace la plus importante à la sécurité de l'Union est antérieure aux attaques terroristes du 11 septembre. Quoi qu'il en soit, ce dernier événement est devenu central dans les changements apportés en Europe à cet espace punitif, en faisant taire les critiques.

En effet, depuis le 11 septembre, les services de renseignement, le G8, l'OTAN, les responsables JAI et du deuxième pilier de l'Union Européenne, Europol dans une moindre mesure, ont eu tendance à s'aligner sur une criminalisation en bloc sur le plan rhétorique, via un transfert progressif d'illégitimité touchant non seulement les membres des réseaux Al-Qaida - ce qui s'impose -, mais aussi de manière plus insidieuse, les islamistes, les personnes venant du Moyen-Orient, les basques radicaux, les membres des organisations de soutien aux kurdes. Les décisions-cadre en matière de terrorisme ont étendu la notion au-delà du raisonnable comme l'a souligné l'organisation Statewatch et comme le reconnaissent dans des entretiens les spécialistes de la lutte anti-terroriste. Mais on ne s'est pas arrêté là, via des rhétoriques oscillant entre la déclaration et les propositions de changement des normes de droit, on a assimilé au terrorisme, au crime et aux atteintes à la sécurité intérieure, l'ensemble des immigrés extra-communautaires placés en situation d'illégalité, les "irréguliers » qu'ils soient migrants ou demandeurs d'asile, les "passeurs » et leurs victimes, les jeunes vivant dans les quartiers difficiles quelle que soit leur nationalité, les usagers d'Internet utilisant des moyens cryptés, et même les contestataires des sommets lorsque ces contestataires usent de moyens radicaux pour faire entendre leur voix. Les textes juridiques en vigueur dans l'Union ont été modifiés dans ce sens, même si les luttes juridiques des ONG et des Cours ont empêché d'atteindre au sein de l'UE l'ampleur des transformations connues aux Etats-Unis. Ils ont enserré les gouvernements au sein de nouvelles règles dont on ne connaît pas encore la jurisprudence. L'acceptation populaire après le 11 septembre a été très forte mais l'instrumentalisation permanente de la peur s'épuise et le doute ainsi que la contestation reprennent. Comprendre que cette contestation n'est pas « un mal qu'il faut combattre " ${ }^{13}$ mais une chance et une opportunité pour l'Union est fondamental. Prendre prétexte de la radicalisation violente pour criminaliser l'ensemble des acteurs sociaux, c'est prendre le risque à terme d'une rupture profonde de légitimité de l'ensemble des institutions, rupture qui dépasse le cadre national et c'est le jeu de certains populismes.

16 Il faut donc étudier les formes de contestation radicalisées - dont l'émergence médiatique avait commencé à Seattle bien qu'elles s'originent dans la lutte anticapitaliste depuis longtemps - et voir comment elles ont pu trouver conjoncturellement des appuis auprès de secteurs sociaux réclamant simplement le droit de circuler et de manifester à l'échelle européenne, en application des textes 
nouvellement promus avec Amsterdam. Ces secteurs sociaux, très divers, et qui ont chacun leur histoire et trajectoire nationale, se sont retrouvés dans les mêmes villes pour dire leur désaccord avec cette «ultra-gouvernementalisation » de la domination transnationale. L'agenda des sommets réunissant les gouvernements a brusquement était récupérée comme leur agenda propre, faisant entrer en résonance, comme sur un pont, la localisation de la domination transnationale représentée par l'espace réservé dans chaque ville aux hôtes puissants et la contestation ouverte des décisions prises dans ces divers sommets qu'ils soient européens ou transnatlantiques. En effet les derniers sommets, dont celui de Gênes est le plus tragique, ont été caractérisés par une tactique des gouvernements visant à utiliser la fragmentation des espaces nationaux contre les contestataires en imposant des restrictions au droit de circuler et au droit de manifester, puis en laissant les institutions de sécurité développer des technologies proactives visant d'abord à bloquer les personnes dès leur départ via des interventions auprès des transporteurs privés ou publics, et ce par «groupe cible » et non plus par individu.

17 Cette forme de refus de la transnationalisation de la contestation comme réponse à la transnationalisation de la domination a conduit à Gênes à des violations manifestes des droits des citoyens considérés par les différentes polices sous l'angle qu'elles avaient institué à l'égard des hooligans. Toute personne voulant circuler avec l'intention déclarée de manifester ou repérée comme " agitateur potentiel » en fonction d'un passé politique inscrit comme dangereux par les services de renseignement, s'est vue alors traitée, individuellement ou collectivement si elle faisait partie d'un groupe, comme hooligan. Cette montée du soupçon liée à l'intransigeance de certains policiers est devenue caricaturale, un peu comme si on interdisait à tout supporter de foot de se déplacer collectivement pour assister à un match, dont on préférerait les gradins vides plutôt que d'avoir un seul acte de vandalisme. Ce blocage en amont de très nombreuses personnes voulant manifester leur désaccord a bien entendu à Nice, à Göteborg et à Gênes, contribué à l'antagonisation entre les forces de sécurité et les protestataires. Surpris les premiers temps, les citoyens et les organisations se sont organisés pour pouvoir voyager et arriver à temps aux sommets, les policiers ont durci leurs stratégies de blocage et ont été amenés à outrepasser en permanence leurs droits concernant le filtrage de qui pouvait légitimement entrer et qui était effectivement déjà un criminel au moins à leurs yeux. Ils ont bloqué les frontières par impossibilité de filtrer efficacement et ont provoqué une crise qu'ils ne peuvent gérer matériellement. En réactivant la technique des «murs", des "périmètres», des «zones», ils ont militarisé leur action et devant l'incapacité d'empêcher les personnes de circuler et d'atteindre la ville du sommet, ils se sont enfermés dans une logique de "théâtre de guerre ». A Québec, à Göteborg, la tactique a été défensive, il s'agissait de protéger une zone et d'empêcher simplement les protestataires d'entrer dans la zone attribuée aux puissants. Il existait de facto deux zones dans la même ville et une frontaliérisation permettant la coexistence du sommet officiel et du sommet alternatif comme l'évoquaient les media. Simplement le succès des contestataires a été dans ces endroits de proposer des discours et un cadre d'action bien plus attractif que celui des officiels.

A Gênes, et c'est là sans doute une des particularités de ce sommet, il a été clair, dès le départ, que les dirigeants voulaient que ce sommet soit le leur et non celui des alternatifs. Les décisions d'utiliser et de renforcer le blocage en amont des individus, de militariser la zone rouge et surtout d'intervenir activement dans les autres zones pour empêcher la tenue d'un sommet alternatif avec l'usage de multiples mini-provocations 
(hélicoptères très bas, empêchant les débats du sommet alternatif d'avoir lieu...) sont de la responsabilité de l'ensemble des dirigeants européens et du G8. Ceci s'est combiné avec la situation politique italienne intérieure depuis l'arrivée de Berlusconi et l'axe que ce dernier voulait créer avec les Etats-Unis en détrônant le Royaume-Uni dans le rôle d'allié le plus fidèle de "George Bush ». Les dirigeants européens, si fermes à l'égard de Heider en Autriche, ont volontiers fermé les yeux sur l'arrivée de Berlusconi. Il faudrait sans doute une commission d'enquête sur Gênes, mais il ne faudrait pas s'arrêter aux conditions nationales dans cette enquête, il faudrait regarder comment les dirigeants de l'Union ont avalisé, voire encouragé l'émergence de telles pratiques avec leur politique des deuxième et troisième piliers, avec leurs déclarations sur l'asile, la migration, le franchissement des frontières. Le groupe Trévi II institué dans le milieu des années 1980 pour des échanges de technologies policières face au radicalisme a-t-il échoué à convaincre les Italiens ou est-il au contraire à l'origine de ce que nous venons de voir?

L'ultra-gouvernementalisation et les résistances qu'elle va engendrer nécessairement, ne peut être une ligne politique tenable pour les élites mondialisées. Elle débouche sur une militarisation des pratiques policières et un déni du judiciaire et du juridique. Il est peu probable que la guerre sociale mondiale soit pour demain. Il n'empêche que la transformation des citoyens contestataires en ennemis militaires, dès qu'ils transnationalisent leurs actions et répondent à la transnationalisation des décisions, a été essayé à Gênes. On a voulu dissuader les jeunes de retourner protester en leur montrant la violence brute et nue. On a voulu faire peur aux pacifistes et on cherchera sans doute à les séparer de leurs branches les plus activistes. Y a-t-on réussi ? Espérons que non. Il y va de la démocratie et des libertés publiques à l'intérieur de l'Union. Les droits de circuler et de manifester à l'échelle transnationale sont les pendants de la transnationalisation des décisions. Ils sont, bien plus encore que l'harmonisation juridique des législations, ce qui fera que l'Europe existera un jour comme territoire. Rappelons-nous des leçons de la formation des Etats et du fait que la construction par les élites ne fait pas l'Etat tant qu'elle n'a pas rencontré les dynamiques sociales de la contestation $^{14}$.

\section{NOTES}

1. . Roland Bleiker, Popular Dissent, Human Agency and Global Politics, Cambridge University Press, 2000.

2. . Susan Strange, The Retreat of the State: The Diffusion of Power in the World Economy, Cambridge, Cambridge University Press, 1996 et Mad Money, Manchester, Manchester University Press, 1998. Voir également Randall Germain, The International Organization of Credit : states and global finance in the world- economy, Cambridge, Cambridge University Press, 1997.

3. . Murray Edelman, Constructing the Political Spectacle, Chicago, University of Chicago Press, 1988. Edition française : Pièces et règles du jeu politique, coll. La couleur des idées, ed. du Seuil, 1991. 
4. . Didier Bigo, Polices en réseaux, l'expérience européenne, Pressesde la Fondation nationale des sciences politiques, Paris, 1996.

5. . Elspeth Guild, Moving the borders of Europe, University of Nijmegen, 97 p. ; Riva Kastoryano, Negotiating Identities. States and Immigrants in France and Germany, Princeton, Princeton University Press, 2002.

6. . Voir « Sociologie de l'Europe », sous la direction de Virginie Guiraudon, Cultures \& Conflits, n³8-39, Paris, L'Harmattan, été-automne 2000.

7. . Voir la première partie du dossier sur Tampere.

8. . Voir Didier Georgakakis, «La démission de la Commission européenne : scandale et tournant institutionnel » (octobre 1998-mars 1999), Cultures \& Conflits, n³8-39, op.cit. 9. . Voir la lettre des Etats-Unis à la Commission européenne, datée du 16 octobre 2001. Le texte est reproduit dans : http://www.statewatch.org/news/2002/feb/useu.pdf 10. . Ayse Ceyhan et Anastassia Tsoukala, «Contrôle de l'immigration : mythes et réalités ", Cultures \& Conflits, n²6/27, Paris, L'Harmattan, été/automne, 1997 ; Elspeth Guild, " The inexpected victims of september eleven. Imigration and asylum ", in Walker Rob et Gokaï Bulent, 11 september 2001 : world, terror and judgement, Ashgate, 2002.

11. Cette conclusion est étayée par la faiblesse du nombre de poursuites criminelles de trafiquants au sein même de l'Union. Lorsque les chiffres sont comparés à ceux des poursuites contre les membres d'importants réseaux de trafic de drogue, la différence est surprenante.

12. Comme cela se dégageait déjà des premiers éléments voir Didier Bigo in Lequesne C. et de La Serre F., Quelle Union pour quelle Europe ?, Bruxelles, Complexe, 1998.

13. . Discours de Berlusconi après Gênes.

14. Ch. Tilly, S. Tarrow, D. Mac Adam, Dynamics of Contention, Cambridge, 2001 ; S. Tarrow, Power in Movement, Cambridge, 1998 ; « Emeutes urbaines : le retour du politique », Cultures \& Conflits, n5, Paris, L'Harmattan, printemps 1992.

\section{RÉSUMÉS}

Recent transnational trends in governmental cooperation are criticised. Politicians at various summits make policies and decisions influenced by governments. Outcomes of several G7, G8, and EU summits are evaluated. Alternative "counter-summits" - e.g., at the WTO meeting in Seattle have attempted to create spaces for popular dissent. These protests have themselves become transnational. Officials systematically and forcibly blocked one at Genoa. Transnational rights of association and demonstration must accompany transnational decision-making, if democracy and liberty are to endure.

\section{INDEX}

Index géographique : Union européenne

Mots-clés : biopouvoir, construction européenne, régionalisme et régionalisation, Relations Internationales 